\title{
SOME UPPER BOUNDS FOR THE DIMENSION OF THE $c$-NILPOTENT MULTIPLIER OF A PAIR OF LIE ALGEBRAS
}

\author{
HOMAYOON ARAByani \\ Mohammad JaVad SADEghifard \\ Department of Mathematics \\ Neyshabur Branch, Islamic Azad University \\ Neyshabur, Iran \\ e-mail: arabyani.h@gmail.com \\ h.arabyani@iau-neyshabur.ac.ir \\ math.sadeghifard85@gmail.com \\ AND \\ Sedighen Sheikh-Mohseni \\ Higher Education Center of Eghlid, Eghlid, Iran \\ e-mail: sh.mohseni.s@gmail.com
}

\begin{abstract}
The notion of the Schur multiplier of a Lie algebra $L$ was introduced by Batten in 1996. Recently, the first author introduced the concept of the $c$ nilpotent multiplier of a pair of Lie algebras and gave some exact sequences for the $c$-nilpotent multiplier of a pair of Lie algebras. The purpose of this paper is to derive some inequalities for dimension of the $c$-nilpotent multiplier of a pair of Lie algebras.
\end{abstract}

Keywords: pair of Lie algebras, Schur multiplier, nilpotent Lie algebra.

2010 Mathematics Subject Classification: 17B30, 17B60, 17B99.

\section{REFERENCES}

[1] H. Arabyani, Bounds for the dimension of the c-nilpotent multiplier of a pair of Lie algebras, Bull. Iranian Math. Soc. 43 (2017) 2411-2418.

doi:10.1142/S1793557119500074 
[2] H. Arabyani, F. Saeedi, M.R.R. Moghaddam and E. Khamseh, Characterization of nilpotent Lie algebras pair by their Schur multipliers, Comm. Algebra 42 (2014) 5474-5483. doi:10.1080/00927872.2012.677081

[3] H. Arabyani and H. Safa, Some properties of c-covers of a pair of Lie algebras, Quaest. Math. 42 (2019) 37-45. doi:10.2989/16073606.2018.1437482

[4] H. Arabyani, Some results on the c-nilpotent multiplier of a pair of Lie algebras, Bull. Iranian Math. Soc. 45 (2019) 205-212. doi:10.1007/s41980-018-0126-6

[5] P. Batten, K. Moneyhun and E. Stitzinger, On characterizing nilpotent Lie algebras by their multipliers, Comm. Algebra 24 (1996) 4319-4330. doi:10.1080/00927879608825817

[6] G. Ellis, Nonabelian exterior products of Lie algebras and an exact sequence in the homology of Lie algebras, J. Pure Appl. Algebra 46 (1987) 111-115. doi:10.1016/0022-4049(87)90089-2

[7] G. Ellis, The Schur multiplier of a pair of groups, Appl. Categ. Structures 6 (1998) 355-371. doi:10.1023/A:1008652316165

[8] G. Karpilovsky, The Schur Multiplier (Clarendon Press, Oxford, 1987).

[9] E.I. Marshal, The Frattini subalgebra of a Lie algebra, J. London Math. Soc. 42 (1967) 416-422. doi:10.1112/jlms/s1-42.1.416

[10] K. Moneyhun, Isoclinisms in Lie algebras, Algebras Groups Geom. 11 (1994) 9-22.

[11] F. Saeedi, H. Arabyani and P. Niroomand, On dimension of Schur multiplier of nilpotent Lie algebra II, Asian-Eur. J. Math. 10 (4) (2017) 1750076 (8 pages). doi:10.1142/S1793557117500760

[12] F. Saeedi, A.R. Salemkar and B. Edalatzadeh, The commutator subalgebra and Schur multiplier of a pair of nilpotent Lie algebras, J. Lie Theory 21 (2011) 491-498.

[13] H. Safa and H. Arabyani, On c-nilpotent multiplier and c-covers of a pair of Lie algebras, Comm. Algebra 45 (2017) 4429-4434.

doi:10.1080/00927872.2016.1265125

[14] A.R. Salemkar and S. Alizadeh Niri, Bounds for the dimension of the Schur multiplier of a pair of nilpotent Lie algebras, Asian-Eur. J. Math. 5 (2012) 1250059 (9 pages). doi:10.1142/S1793557112500593

[15] A.R. Salemkar, B. Edalatzadeh and M. Araskhan, Some inequalities for the dimension of the c-nilpotent multiplier of Lie algebras, J. Algebra 322 (2009) 1575-1585. doi:10.1016/j.jalgebra.2009.05.036

[16] I. Schur, Über die Darstellung der endlichen Gruppen durch gebrochene lineare Substitutionen, J. Reine Angew. Math. 127 (1904) 20-50. doi:10.1515/crll.1904.127.20

Received 31 August 2019

Revised 9 December 2019

Accepted 10 December 2019 